TITLE:

\title{
Dynamic hysteresis modeling of silicon steel having nonuniform magnetic property
}

$\operatorname{AUTHOR}(\mathrm{S})$ :

Mitsuoka, R.; Mifune, T.; Matsuo, T.

\section{CITATION:}

Mitsuoka, R. ... [et al]. Dynamic hysteresis modeling of silicon steel having nonuniform magnetic property. JOURNAL OF APPLIED PHYSICS 2012, 111(7): 07 D110.

\section{ISSUE DATE:}

2012-02

URL:

http://hdl.handle.net/2433/160647

\section{RIGHT:}

Copyright 2012 American Institute of Physics. This article may be downloaded for personal use only. Any other use requires prior permission of the author and the American Institute of Physics. The following article appeared in JOURNAL OF APPLIED PHYSICS 111, 07D110 (2012) and may be found at http://link.aip.org/link/?jap/111/07D110 


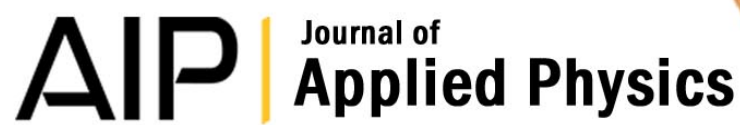

\section{Dynamic hysteresis modeling of silicon steel having nonuniform magnetic property}

R. Mitsuoka, T. Mifune, and T. Matsuo

Citation: J. Appl. Phys. 111, 07D110 (2012); doi: 10.1063/1.3672864

View online: http://dx.doi.org/10.1063/1.3672864

View Table of Contents: http://jap.aip.org/resource/1/JAPIAU/v111/i7

Published by the American Institute of Physics.

\section{Related Articles}

The effect of interfaces on magnetic activation volumes in single crystal Co2FeSi Heusler alloy thin films Appl. Phys. Lett. 101, 102410 (2012)

Pulse voltage-induced dynamic magnetization switching in magnetic tunneling junctions with high resistancearea product

Appl. Phys. Lett. 101, 102406 (2012)

Magnetism in MoS2 induced by proton irradiation

Appl. Phys. Lett. 101, 102103 (2012)

On physical aspects of the Jiles-Atherton hysteresis models

J. Appl. Phys. 112, 043916 (2012)

Spin current generator based on topological insulator coupled to ferromagnetic insulators

AlP Advances 2, $032162(2012)$

\section{Additional information on J. Appl. Phys.}

Journal Homepage: http://jap.aip.org/

Journal Information: http://jap.aip.org/about/about_the_journal

Top downloads: http://jap.aip.org/features/most_downloaded

Information for Authors: http://jap.aip.org/authors

\section{ADVERTISEMENT}

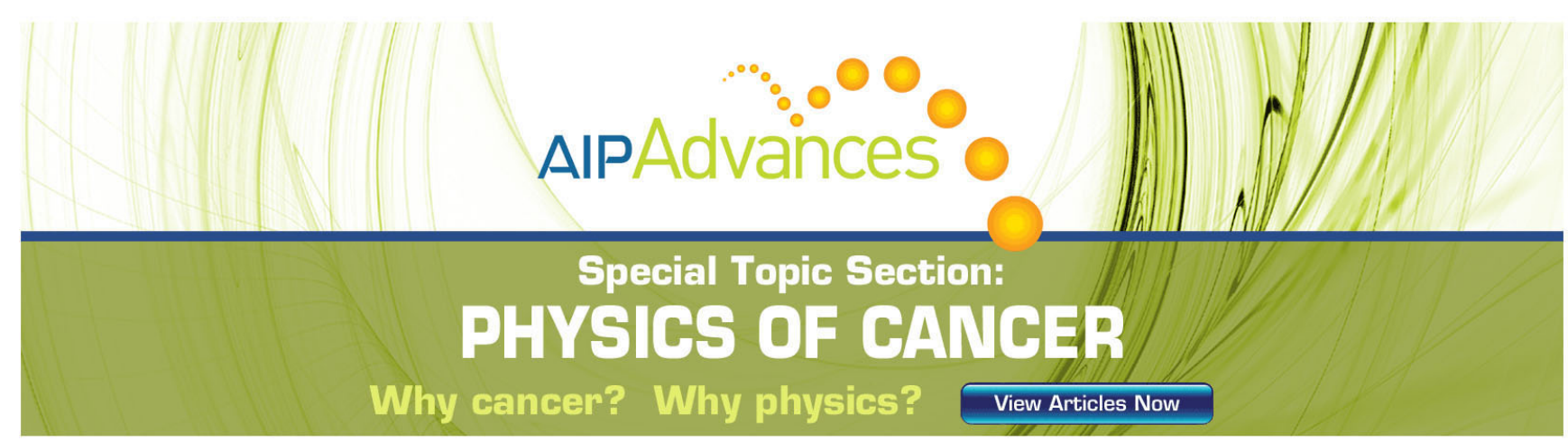




\title{
Dynamic hysteresis modeling of silicon steel having nonuniform magnetic property
}

\author{
R. Mitsuoka, T. Mifune, and T. Matsuo ${ }^{\text {a) }}$ \\ Department of Electrical Engineering, Kyoto University, Kyoto, Japan
}

(Presented 3 November 2011; received 23 September 2011; accepted 28 October 2011; published online 24 February 2012)

\begin{abstract}
Finite-element eddy-current analysis is combined with the play model to represent the AC hysteretic properties of a silicon steel sheet. The dependence of the anomaly factor on the excitation frequency is discussed. The finite element analysis with a constant anomaly factor overestimates the iron loss relative to the result obtained from classical eddy-current theory because of the nonlinearity of magnetization. The nonuniform magnetic property along the thickness direction is examined, and a dynamic anomaly factor is then introduced to directly represent the frequency dependence. The alternating magnetic properties simulated by the proposed model agree well with measured properties. (C) 2012 American Institute of Physics. [doi:10.1063/1.3672864]
\end{abstract}

\section{INTRODUCTION}

The accurate evaluation of iron loss for a silicon steel sheet requires the computation of both hysteresis and eddycurrent losses in the presence of the skin effect. To take the skin effect into account, several dynamic hysteresis models $^{1,2}$ require a one-dimensional sub-analysis along the thickness direction. A computational study ${ }^{3}$ has shown that a nonlinear magnetic property may yield a larger eddy-current loss than a linear property. The results of the present study suggests that discrepancies between the measured and calculated iron losses are due to the magnetic property being assumed uniform rather than nonuniform along the thickness direction.

This paper first discusses the effect of the nonuniform magnetic property along the thickness direction on the eddycurrent loss because magnetization is likely to proceed nonuniformly, as discussed in Ref. 4. Next, a dynamic anomaly factor is introduced to represent the dependence of the anomalous eddy-current loss on the excitation frequency. ${ }^{5}$

\section{FINITE ELEMENT ANALYSIS OF IRON LOSS}

To compare with the simulated iron loss, the magnetic properties of a non-oriented silicon steel (JIS 35A230 grade) sheet are measured under a condition of alternating sinusoidal magnetic flux. The measured eddy-current loss, $L_{\text {ed }}$, is compared with the classical eddy-current loss, ${ }^{4} L_{\mathrm{cl}}$ $=\pi \omega \sigma d^{2} B_{\mathrm{a}}^{2} / 12$, where $\omega$ is the angular frequency, $B_{\mathrm{a}}$ is the amplitude of the magnetic flux density, $\sigma=1.818 \times 10^{6} \mathrm{~S} / \mathrm{m}$ is the conductivity, and $d=0.35 \mathrm{~mm}$ is the sheet thickness. Figure 1 (a) shows the anomaly factor, $k=L_{\mathrm{ed}} / L_{\mathrm{cl}}$, where $k$ is larger than 1 because of the anomalous eddy-current loss. ${ }^{4}$ The anomaly factor decreases with an increase in frequency, whereas the effect of $B_{\mathrm{a}}$ is small. The previously mentioned frequency dependence of $k$ is possibly due to the skin effect, because the skin depth becomes comparable to the sheet thickness when the frequency becomes high.
To evaluate the skin effect, a one-dimensional finite element (FE) eddy-current analysis is carried out along the thickness direction with the play model. ${ }^{6}$ The play model is an efficient static hysteresis model whose properties are equivalent to those of the classical Preisach model. ${ }^{4}$ To represent the anomalous eddy-current loss, the conductivity is multiplied by a constant, $k=1.6$, which is the averaged anomaly factor at $50 \mathrm{~Hz}$. Figure 1(b) compares the iron loss per cycle simulated by the FE model with that obtained from classical eddy-current theory. The iron loss given by the FE model is greater than that given by the classical eddy-current model, despite the presence of the skin effect in the FE model. It is computationally confirmed that the greater iron loss simulated in the FE analysis is observed even when the hysteretic property is neglected. This suggests that the frequency dependence of the anomaly factor is not due to the skin effect mainly when the frequency is less than $500 \mathrm{~Hz}$. The greater iron loss simulated in the FE analysis is probably due to nonlinear magnetization, as is discussed in the following section. Section III discusses the effect of the nonuniformity of the magnetization property along the thickness direction on the eddy-current loss, which is a possible reason for the discrepancy between the measured and calculated iron losses.

\section{EFFECT OF NONUNIFORM PROPERTY}

As was discussed in Ref. 4, magnetization is likely to proceed nonuniformly in magnetic materials, which affects the anomalous eddy-current loss. This section discusses the effect of the nonuniform magnetic property along the thickness direction on the eddy-current loss.

\section{A. Linear magnetization model}

First, the effect of the nonuniformity is discussed for the linear magnetization as follows.

The magnetic field is written as

$$
H(z)=H_{0}+H_{\mathrm{e}}(z),
$$

${ }^{a)}$ Electronic mail: tmatsuo@kuee.kyoto-u.ac.jp. 


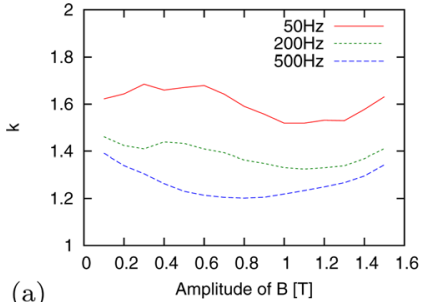

(a) Amplitude of $B$ [T]

(b)

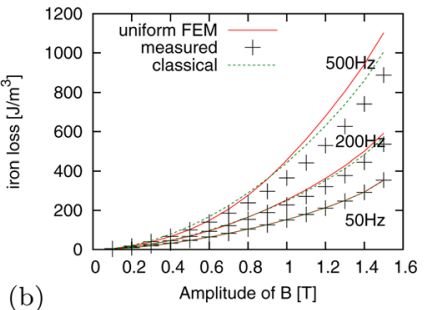

FIG. 1. (Color online) Iron-loss properties: (a) anomaly factor, and (b) iron loss per cycle simulated with constant, $k$.

where $H_{0}$ is the applied magnetic field, $H_{\mathrm{e}}$ is the magnetic field induced by the eddy current, and the $z$-direction is the thickness direction. It is assumed that the magnetic field is along the $x$-direction and that $H_{\mathrm{e}} \ll H_{0}$.

The eddy-current field is described by

$$
\begin{gathered}
H_{\mathrm{e}}(z)=\int_{d / 2}^{z} \sigma\left(z^{\prime}\right) E\left(z^{\prime}\right) d z^{\prime}, \\
E(z)=\int_{0}^{z} \mu\left(z^{\prime}\right) \frac{\partial H_{0}}{\partial t} d z^{\prime},
\end{gathered}
$$

where $E$ is the induced electric field along the $y$-direction, $\mu$ is the permeability, and $\sigma$ is the effective conductivity. The effective conductivity is the product of the anomaly factor and the conductivity of silicon steel, where the spatial dependence of the anomaly factor due to the nonuniform magnetic domain structure is taken into account. It is assumed that $\sigma$ and $\mu$ are $\sigma=\sigma_{0} f(z)$ and $\mu=\mu_{0} g(z)$, respectively, where the averaged values of $f(z)$ and $g(z)$ are 1 .

From Eq. (2), $H_{\mathrm{e}}$ is given as

$$
H_{\mathrm{e}}(z)=\mu_{0} \sigma_{0} \int_{d / 2}^{z} f\left(z^{\prime}\right)\left[\int_{0}^{z^{\prime}} g\left(z^{\prime \prime}\right) d z^{\prime \prime}\right] d z^{\prime} \frac{\partial H_{0}}{\partial t} .
$$

The average flux density $B_{0}$ is approximated as

$$
B_{0} \simeq \mu_{0}\left(H_{0}+H_{\text {ea }}\right),
$$

where $H_{\text {ea }}$ is the average of $H_{\mathrm{e}}$, which is given as

$$
H_{\mathrm{ea}} \simeq \sigma_{0} \frac{2}{d} \int_{0}^{d / 2} \int_{d / 2}^{z} f\left(z^{\prime}\right)\left[\int_{0}^{z^{\prime}} g\left(z^{\prime \prime}\right) d z^{\prime \prime}\right] d z^{\prime} d z \frac{\partial B_{0}}{\partial t} .
$$

From Eq. (5), $H_{0}$ is written as

$$
H_{0}=\frac{B_{0}}{\mu_{0}}-H_{\text {ea }} .
$$

When $g(z)=f(z)=1, H_{0}$ becomes

$$
H_{0}=\frac{B_{0}}{\mu_{0}}+\frac{\sigma_{0} d^{2}}{12} \frac{\partial B_{0}}{\partial t},
$$

which yields the classical eddy-current model.

First, it is assumed that $f(z)=1+a_{\sigma} c(z)$ and $g(z)=1$, where

$$
c(z)=\frac{4}{d}\left(|z|-\frac{d}{4}\right) .
$$

Equations (6) and (7) are reduced to

$$
H_{0}=\frac{B_{0}}{\mu_{0}}+\frac{\sigma_{0} d^{2}}{12}\left(1+\frac{a_{\sigma}}{2}\right) \frac{\partial B_{0}}{\partial t} .
$$

The eddy-current field increases with $a_{\sigma}$ because $H_{\mathrm{e}}$ is induced by $\sigma(z) E(z)$ within $z^{\prime} \leq z \leq d / 2$, according to Eq. (2). Accordingly, the eddy-current field increases when $\sigma(z)$ increases with $|z|$.

When $f(z)=1$ and $g(z)=1+a_{\mu} c(z), H_{0}$ is given as

$$
H_{0}=\frac{B_{0}}{\mu_{0}}+\frac{\sigma_{0} d^{2}}{12}\left(1-\frac{a_{\mu}}{4}\right) \frac{\partial B_{0}}{\partial t} .
$$

The eddy-current field decreases with an increase in $a_{\mu}$ because $E$ is induced by $\mu(z) \partial H_{0} / \partial t$ within $0 \leq z \leq z^{\prime}$, according to Eq. (3). Accordingly, the eddy-current field increases when $\mu(z)$ decreases with $|z|$.

The preceding linear model suggests that the greater iron loss given by the FE model is due to the nonlinear magnetic property. Because of the skin effect, the magnetic field near the surface is stronger than that near the center. Consequently, the permeability decreases with an increase in $|z|$ because of the magnetic saturation. The nonuniform permeability strengthens the eddy-current field. However, the preceding linear model cannot evaluate the skin effect itself, because of the assumption of small $H_{\mathrm{e}}$. The FE analysis for a nonuniform and nonlinear magnetic property is carried out in the following subsection.

\section{B. Nonlinear magnetization model}

\section{Nonuniform magnetic property}

To represent the nonuniform magnetic property along the thickness direction, a simple spatially dependent hysteretic magnetic property is considered,

$$
H(z)=\left[1+a_{\mathrm{h}} c(z)\right] h\left(\left[1+a_{\mathrm{b}} c(z)\right] B(z)\right),
$$

where $h(B)$ is a static hysteretic function represented by the play model; $a_{\mathrm{b}}$ and $a_{\mathrm{h}}$ are the spatially dependent scale factors along the $B$ and $H$ directions, respectively, which do not directly correspond to $a_{\mu}$ in the preceding subsection because of the hysteresis.

Figure 2(a) shows the frequency dependence of the simulated iron loss per cycle given by the nonunifom model with $\left(a_{\mathrm{b}}, a_{\mathrm{h}}\right)= \pm(0.3,0.1)$. When the magnetization is easier near the sheet surface than inside the sheet, the iron loss decreases, as predicted by the linear model. As a result, the
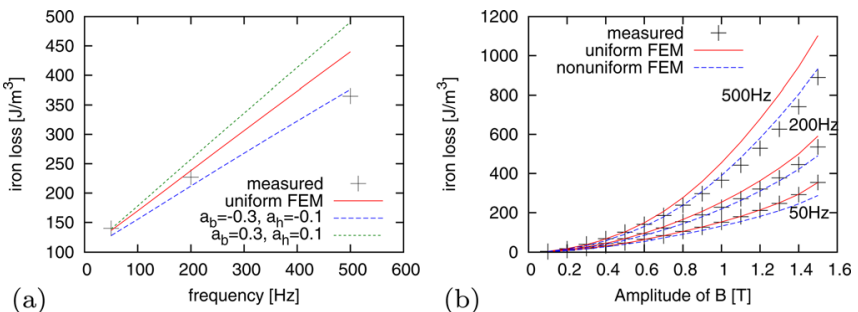

FIG. 2. (Color online) Iron loss per cycle for a nonuniform magnetic property: (a) dependence on frequency at $1 \mathrm{~T}$, and (b) dependence on the amplitude of $B$. 

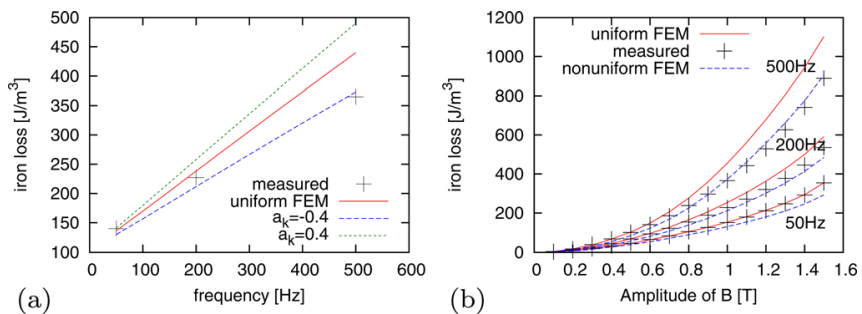

FIG. 3. (Color online) Iron loss per cycle with a nonuniform anomaly factor: (a) dependence on frequency at $1 \mathrm{~T}$, and (b) dependence on amplitude of $B$.

iron loss approximately agrees with the measured loss. Figure 2(b) compares the simulated iron losses per cycle given by the nonuniform model with $\left(a_{\mathrm{b}}, a_{\mathrm{h}}\right)=(-0.3,-0.1)$ and the uniform model with $\left(a_{\mathrm{b}}, a_{\mathrm{h}}\right)=(0,0)$. The nonuniform model improves the iron-loss evaluation at 200 and $500 \mathrm{~Hz}$.

Magnetization, however, is usually not easy near the sheet surface because of the surface magnetic poles, which conflicts with the preceding result. Accordingly, it seems unreasonable that the loss property is explained by the nonuniform magnetic property despite the agreement with the measured property.

\section{Nonuniform anomaly factor}

If the magnetic domain structure is nonuniform along the thickness direction, the anomaly factor, $k$, has spatial dependence. The simple nonuniformity of $k$ is considered similarly to Sec. III A,

$$
k(z)=\left[1+a_{\sigma} c(z)\right] k_{0},
$$

where $k_{0}$ is a constant given by the averaged anomaly factor at $50 \mathrm{~Hz}$.

Figure 3(a) shows the frequency dependence of the simulated iron loss per cycle given by the nonuniform model with $k_{0}=1.6$ and $a_{\sigma}= \pm 0.4$. According to the linear analysis in Sec. III A, the eddy-current loss increases by a factor of $\left(1+a_{\sigma} / 2\right)$, which roughly agrees with the simulation result, on average, even though the linear analysis cannot represent the frequency dependence of $k$. Figure 3(b) compares the simulated iron losses per cycle given by the nonuniform model with $a_{\sigma}=-0.4$ and the uniform model. The nonuniform model improves the iron-loss evaluation.

Figure 4 portrays the $\mathrm{BH}$ loops given by the nonuniform and uniform models with the amplitude, $B_{\mathrm{a}}$, of $0.5,1.0$, and 1.5 T. The nonuniform model yields more accurate $\mathrm{BH}$ loops than the uniform model and the classical eddy-current model.
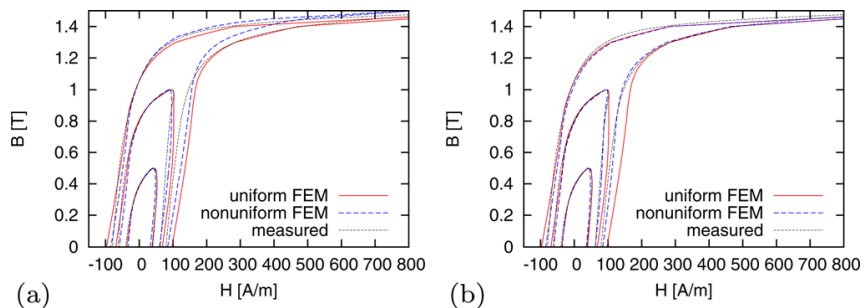

FIG. 4. (Color online) Simulated BH loops at $200 \mathrm{~Hz}$ : (a) with a nonuniform magnetic property, and (b) with a nonuniform anomaly factor.
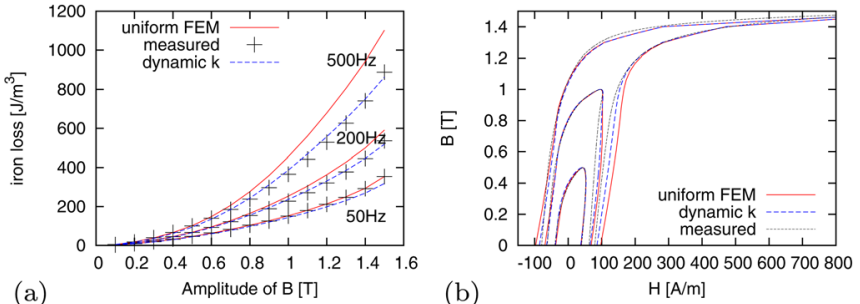

FIG. 5. (Color online) Properties simulated with the dynamic anomaly factor: (a) iron loss per cycle, and (b) BH loops at $200 \mathrm{~Hz}$.

\section{DYNAMIC ANOMALY FACTOR}

Figures 2(a) and 3(a) show that the nonuniform properties do not completely represent the frequency dependence of the skin effect. It is known that the domain wall width of silicon steel decreases with an increase in the magnetization frequency, ${ }^{7,8}$ which decreases $k^{5}$ This section introduces a dynamic anomaly factor to represent the frequency dependence of $k$ phenomenologically without using $f$ explicitly for timedomain simulation as follows:

$$
k=k_{0} \frac{1}{1+\left|\frac{1}{2 \pi f_{0} B_{\mathrm{a} 0}} \frac{\mathrm{d} B}{\mathrm{~d} t}\right|^{\gamma}},
$$

where $\gamma$ represents the frequency dependence of $k, f_{0}$ and $B_{\mathrm{a} 0}$ are constants for normalization, $k_{0}$ is the anomaly factor at $f \ll f_{0}$, whereas $k$ decreases with the increase in $f$ when $f>f_{0}$. According to the measured frequency dependence of $k, k_{0}, \gamma, f_{0}$, and $B_{\mathrm{a} 0}$ are set to $3.7,0.29,40 \mathrm{~Hz}$, and $1.2 \mathrm{~T}$, respectively.

The simulated iron loss per cycle and $\mathrm{BH}$ loops are shown in Fig. 5, where the dynamic anomaly factor gives accurate AC properties.

\section{CONCLUSION}

Nonuniform magnetic properties and a dynamic anomaly factor are discussed to explain the frequency dependence of the anomaly factor of a silicon steel sheet. The latter seems more practical to use than the former because it is difficult to measure the nonuniformity of a magnetic property along the thickness direction.

\section{ACKNOWLEDGMENTS}

This work was supported in part by the Japan Society for the Promotion of Science, Grant-in-Aid for Scientific Research (C), Grant No. 23560328.

${ }^{1}$ O. Bottauscio, M. Chiampi, and D. Chiarabaglio, IEEE Trans. Magn. 36, $561(2000)$

${ }^{2}$ S. E. Zirka et al., IEEE Trans. Magn. 44, 2113 (2008).

${ }^{3}$ E. Dlala, A. Belahcen, J. Pippuri, and A. Arkkio, IEEE Trans. Magn. 46, 306 (2010).

${ }^{4} \mathrm{G}$. Bertotti, Hysteresis in Magnetism (Academic, New York, 1998).

${ }^{5} \mathrm{Y}$. Gao, Y. Matsuo, and K. Muramatsu, "Investigation on simple numeric modeling of anomalous eddy current loss in steel plate using modified conductivity," IEEE Trans. Magn. (to be published).

${ }^{6}$ T. Matsuo and M. Shimasaki, IEEE Trans. Magn. 41, 3112 (2005).

${ }^{7}$ M. R. G. Sharp and J. T. Horner, J. Phys. D: Appl. Phys. 6, 1835 (1973).

${ }^{8}$ J. W. Shilling, IEEE Trans. Magn. 9, 351 (1973). 